\title{
Caracterización de etapas de perforado en probetas multicapas mediante la emisión acústica generada durante la perforación
}

\author{
Characterization of drilling stages in multilayer specimens through the \\ Acoustic Emission generated during the drilling process
}

\author{
Guido Claudio Ferrari ${ }^{1}$, Martín Pedro Gómez ${ }^{2}$ (D)
}

Cómo citar: Ferrari, G.C. y Gómez, M.P. (2020). Caracterización de etapas de perforado en probetas multicapas mediante la emisión acústica generada durante la perforación.. Tecnura, 24(63) 26-39. DOI: https://doi. org/10.14483/22487638.16193

Fecha de de recepción: 31 de mayo de 2019

\section{RESUMEN}

Objetivo: El presente trabajo se basa en el monitoreo mediante emisión acústica de la herramienta de corte en un proceso de perforación de material geológico y la utilización de redes neuronales tipo Kohonen para la clasificación de la información.

Metodología: La metodología consistió en la realización de una serie de perforaciones sobre una probeta construida con rocas tipo arenisca, caliza y pizarra, dispuestas en capas y consolidadas con una mezcla de cemento. El herramental utilizado para la perforación consistió en un cortador de dos filos de carburo de tungsteno (CT), de $65 \mathrm{~mm}$ de diámetro. El proceso completo fue monitoreado mediante un sistema de emisión acústica acoplado a la broca giratoria y a la probeta de ensayo. Posteriormente se correlacionó la emisión acústica con la estratigrafía de la perforación, se procesó y adaptó la información para entrenar y simular una red neuronal tipo Kohonen, que clasificó la información del proceso según el tipo de roca que se estaba atravesado con el cortador.
Fecha de de aceptación: 18 de octubre de 2019

Resultados: Los resultados muestran que la técnica de emisión acústica es sensible a los cambios de estratos durante la perforación de estos geomateriales y que la instrumentación de la broca rotante brinda un buen canal de monitoreo de este proceso. En este se observan los transitorios de cambio de interfaces y el perforado estable mediante el análisis de parámetros de la emisión acústica como RMS, energía MARSE, rise time y frecuencia promedio.

Conclusiones: La técnica de emisión acústica puede ser utilizada para el monitoreo del proceso de taladrado en la presente escala. El procesamiento de los parámetros de emisión acústica permitió entrenar y simular una red neuronal Kohonen que pueda clasificar diferentes etapas del perforado con un error de mezclado inferior al $5 \%$.

Palabras clave: emisión acústica; perforación, monitoreo de procesos; arenisca; caliza; redes neuronales.

1 Ingeniero Mecánico (UTN-FRD). Magíster en Ciencia y Tecnología, mención Materiales (IT-UNSAM-CNEA). Docente de la Universidad Tecnológica Nacional - Facultad Regional Delta de Campana, Buenos Aires, Argentina. Contacto: guicferrari@gmail.com

2 Licenciado en Física, magíster en Ciencia y Tecnología de los Materiales, doctor en Ciencia y Tecnología de los Materiales. Comisión Nacional de Energía Atómica. Docente de la Universidad Tecnológica Nacional Regional Delta y de la Universidad Nacional de San Martín. Ciudad Autónoma de Buenos Aires, Argentina. Contacto: mpgomez@cnea.gov.ar

ORCID: http://orcid.org/0000-0001-9578-2399 


\section{ABSTRACT}

Objective: This work is based on the Acoustic Emission (EA) monitoring of the cutting tool in a geological material drilling process and the use of Kohonen type Neural Networks for the classification of information.

Methodology: The methodology consisted in the realization of a series of perforations on a specimen built with sandstone, limestone and slate rocks, arranged in layers and consolidated with a cement mixture. The tool used for drilling consisted of a double-edged Tungsten Carbide (CT) cutter, $65 \mathrm{~mm}$ in diameter. The entire process was monitored by an AE system coupled to the rotating drill and test specimen. Subsequently, the EA was correlated with the stratigraphy of the perforation, the information was processed and adapted to train and simulate a Kohonen type neural network, which classified the process information according to the type of rock that was traversed with the cutter.
Results: The results show that the Acoustic Emission technique is sensitive to stratum changes during the drilling process of these geomaterials and that the instrumentation of the rotating driII provides a good monitoring channel for this process. In this, the change of interfaces of the interfaces and the stable drilling process can be observed through the analysis of acoustic emission parameters such as rms, MARSE energy, rise time and average frequency.

Conclusions: The Acoustic Emission technique can be used to monitor the drilling process on this scale. The processing of the Acoustic Emission parameters allowed to train and simulate a Kohonen neural network that can classify different stages of the drilling process with a mixing error of less than $5 \%$.

Keywords: Acoustic Emission; Drilling; Process Monitoring; Sandstone; Neural Networks.

\section{INTRODUCCIÓN}

La técnica de emisión acústica (EA) es ampliamente utilizada como una valiosa herramienta en ensayos no destructivos para el control, detección y posicionamiento de diferentes defectos en recipientes a presión, tanques de almacenamiento de hidrocarburos, pérdidas en válvulas y cañerías, descargas tipo corona en transformadores de alta tensión, rotura de fibra y matriz de materiales compuesto tipo FVRP y FCRP, análisis de propagación de fracturas en hormigón, etc. Esta técnica consiste en la detección y análisis de las ondas elásticas resultantes de la liberación de energía durante un corto periodo de tiempo, donde la emisión proviene de una fuente (o suceso) que es excitada de alguna manera. Ejemplo de este fenómeno es la liberación de energía potencial elástica durante el avance de una fractura frágil. Parte de esa energía liberada por la fisura se propaga a través del cuerpo fracturado en forma de onda de tensión elástica, donde eventualmente llega a un transductor piezoeléctrico que permite adquirir la señal de EA para su registro y posterior análisis. En particular, la propagación de una fractura se caracteriza por ser una emisión de tipo discreta, es decir temporalmente determinada.

Otra forma de utilizar la EA está relacionada con el monitoreo indirecto de procesos; con ellas se evalúa el desarrollo y evolución, por ejemplo, del corte de un material respecto de variables como el avance, velocidad de corte, material y condición del herramental, entre otras. Este tipo de EA, al igual que los procesos de fricción y erosión, genera señales que no están claramente determinadas en función del tiempo y tienden más bien a ser continuas con transitorios a lo largo del proceso. Dentro de la caracterización de las señales de EA se 
pueden parametrizar diferentes aspectos de la onda recibida como la duración, la amplitud máxima, el número de cuentas, la energía MARSE, la frecuencia promedio, el centroide de frecuencia, el tiempo de excursión hasta la amplitud máxima (denominado rise time) y el valor RMS de la EA, entre otros parámetros. Algunos de estos valores se calculan a partir del momento que la señal sobrepasa un valor arbitrario denominado umbral, el cual es fijado por el operador del equipo en función de su conocimiento y experiencia.

Existen trabajos que analizan el monitoreo de procesos de corte ortogonal, por ejemplo en metales, donde se asocia el RMS de la señal de EA con ciertas condiciones durante el torneado. Dornfeld y Kannatey-Asibu (1980), y Saini y Park (1996), generaron modelos empíricos y analíticos asociando este valor (RMS de EA) en función de variables como deformación plástica del material cortado, flujo de viruta, avance y r. p. m. Otros análisis, como el de Grabec y Leskovar (1977), también incluyen distribución del espectro de frecuencia de la señal de EA respecto del avance, $r$. p. m. y profundidad de corte. Jemielniak y Otman (1997) estudian el análisis de la curtosis y oblicuidad (skewness) de la distribución del parámetro RMS de EA, donde se muestra una buena sensibilidad para detectar la rotura catastrófica de la herramienta.

Para el taladrado de materiales compuestos en fibra de carbono y resina epoxi, Mascaro, Gibiat, Bernadou y Esquerre (2005) relacionan el espectro de frecuencia durante este proceso y muestran que la banda de frecuencia concerniente a la rotura de la matriz y de las fibras no varía con el cambio de geometría del herramental, la velocidad de taladrado y el avance. Además, indican que la energía de la señal de la EA es sensible al desgaste de la herramienta, lo que revela que eventualmente este parámetro podría ser utilizado para el control de su estado. Trabajos más recientes utilizan parámetros secundarios como la potencia media de la EA, los cuales funcionan como indicadores del estado de las brocas en perforados de metales (Gómez, Hey, Ruzzante y D’Attellis, 2010; Gómez 2012).

La perforación, desde un punto de vista mecánico, es un proceso complejo de modelar debido a la cantidad de variables que se deben considerar, a saber: geometría del cortador (Rajabov et al., 2012), cantidad de filos, tipo de mecanismo de corte, desintegración del material, forma de remoción, lubricación, temperatura, r. p. m., fuerza de avance (FA), etc. Se deben considerar también las características del material a ser cortado, que modifica la interacción herramienta/material en perforación de materiales rocosos (Bourgoyne et al., 1991) y, por tanto, también produce una modificación de la dinámica del proceso de corte relacionada con el tipo de material, constantes elásticas, fragilidad, ductilidad, dureza, granulometría, densidad, nivel de saturación de líquido y condiciones de tensión del ambiente que lo rodea, ya que diferentes entornos de confinamiento (equiaxial, poliaxial, nivel de tensión deviatoria, etc.) modifican el comportamiento ante la fractura para un mismo material (Peng y Zhang, 2007).

La información obtenida durante el monitoreo de procesos es cuantiosa. Para poder representar la evolución de estos procedimientos estocásticos es necesario el registro de grandes volúmenes de información donde la utilización de parámetros es una forma económica de resumir el comportamiento de la señal, siempre a coste de perder información. Los parámetros de EA sujetos a análisis pueden ser varios, y deben ser seleccionados en función del peso que tienen a la hora de describir el proceso que se desea monitorear. Es una práctica recomendada adicionar a los parámetros de la EA alguna variable adicional perteneciente al control del proceso, como por ejemplo la variación de la fuerza 
de avance en el perforado, la temperatura, la presión, u otro indicador dinámico para correlacionar la información de la EA. Esto determina que la cantidad de variables que se debe manejar sea alta y convierta al análisis de la condición de proceso en un problema de características multiparamétrico, en donde los puntos máximos o mínimos del sistema se encuentran posiblemente en combinaciones no lineales entre estas variables.

Allí la matemática, los métodos de cálculo actuales y la tecnología se conjugan para proveer formas de análisis que reúnen varias de las características necesarias para modelar estos sistemas (Everson y Cheraghi, 1999; Philippidis, Nikolaidis y Anastassopoulos, 1998).

Para el presente trabajo, se utiliza una red neuronal de tipo Kohonen o mapa autoorganizado (SOM, por su sigla en inglés) el cual tiene la capacidad de crear una grilla planar (mapa) con neuronas que se agrupan para clasificar diferentes tipos de información según reglas de aprendizaje específicas.

Este tipo de red fue utilizada con éxito en diferentes aplicaciones, como la detección de fisuras en fabricación de mosaicos (Barrera, Carreón y Ruiz, 2005); clasificación de origen de aceites de oliva y medición de la reactividad de enlaces químicos (Zupan y Gasteiger, 1993); mejora de las características de las señales (relación señal ruido) de la EA en el control de desgaste de herramientas de micro maquinado (Chen y Liao, 2003); análisis de modos de falla en materiales compuestos (Godin, Huguet y Gaertner, 2005); tratamiento de imágenes y señales en general; entre otras variadas aplicaciones (Kohonen, 2001).

\section{METODOLOGÍA}

Sistema de perforación: El sistema de perforación posee un equipo hidráulico con regu- lación de avance de 120 bar de potencia, con $1000 \mathrm{~mm}$ de recorrido y propulsado con un motor de 4 hp trabajando hasta 3000 r. p. m., el cual está conectado a una caja reductora y a un variador de velocidad para controlar la velocidad de rotación de la broca (figura 1a). El herramental de perforación se compone de cuerpo tubular de $1600 \mathrm{~mm}$ de longitud, donde en su extremo se fija la broca de perfo-ración (figura 1b). de 65 mm diámetro con dos filos de carburo de tungsteno y un ángulo de punta de $162^{\circ}$. Adicionalmente, se cuen-ta con sistema de inyección de agua para la lubricación y extracción de material cortado. Este prototipo está adaptado para instrumen-tar directamente la broca de perforación (he-rramienta rotante) y obtener la señal de EA procedente del proceso de corte que se desa-rrolla en el extremo del cortador.

Probetas de material multicapa: Las probetas ensayadas son paralelepípedos de $320 \times 320 \mathrm{~mm}$ de base y $400 \mathrm{~mm}$ de altura, compues-tas por capas de material geológico (rocas) de diferentes características colocadas en forma de estratos (figura 2a). Una vez definidos es-tos estratos (capas de rocas), se cementaron con material en una proporción 3:0,8:0,2 de arena, cemento y cal, respectivamente. Estas unidades consolidadas conforman una mues-tra de ensayo sobre la cual se pueden realizar 4 perforaciones simétricas, como se observa en la figura $2 \mathrm{~b}$. Además, cada una de la muestras fue instrumentada con una guía de ondas con el fin de obtener la EA del proceso de perforación por un camino alternativo al de la columna de perforación y comparar así su desempeño.

Las probetas construidas de esta manera pre-sentan gran heterogeneidad geométrica respec-to del espesor de cada estrato, así como en el tallado para la conformación de cada capa (fi-gura $3 a$, b y

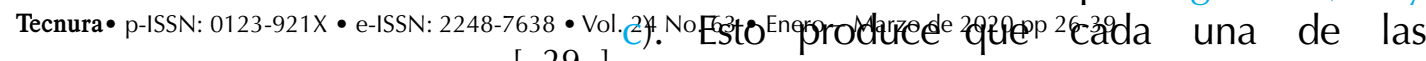
[ 29 ] probetas y de las perforaciones realizadas sean únicas e irrepetibles 
En la tabla 1. se detallan las composiciones de los dos tipos de probetas ensayadas para el presente trabajo. La muestra M1 contiene dos tipos de rocas sedimentarias de diferente tamaño de grano y material constituyente. El primer estrato pertenece a un tipo de roca a.

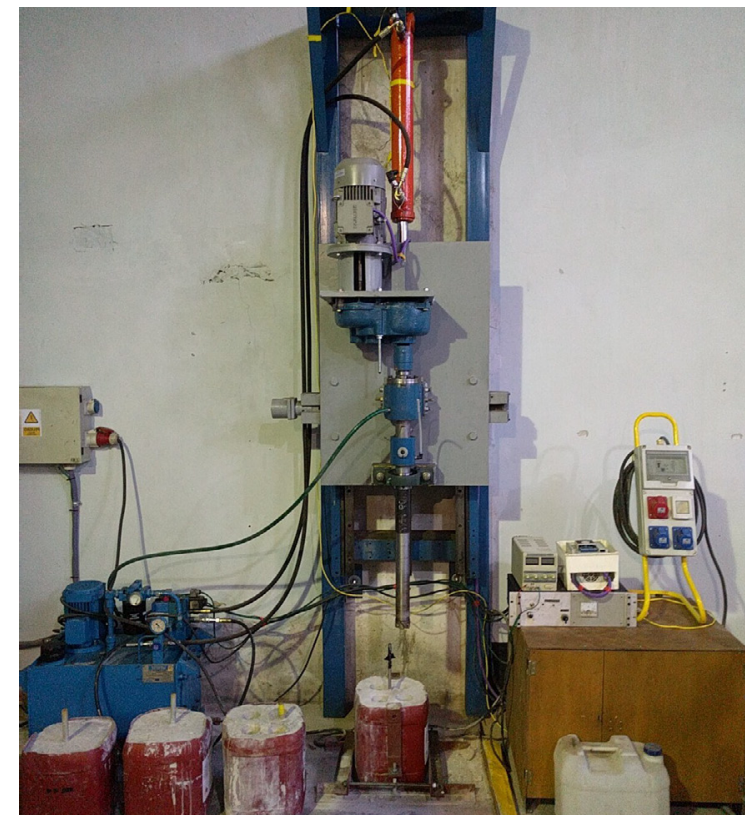

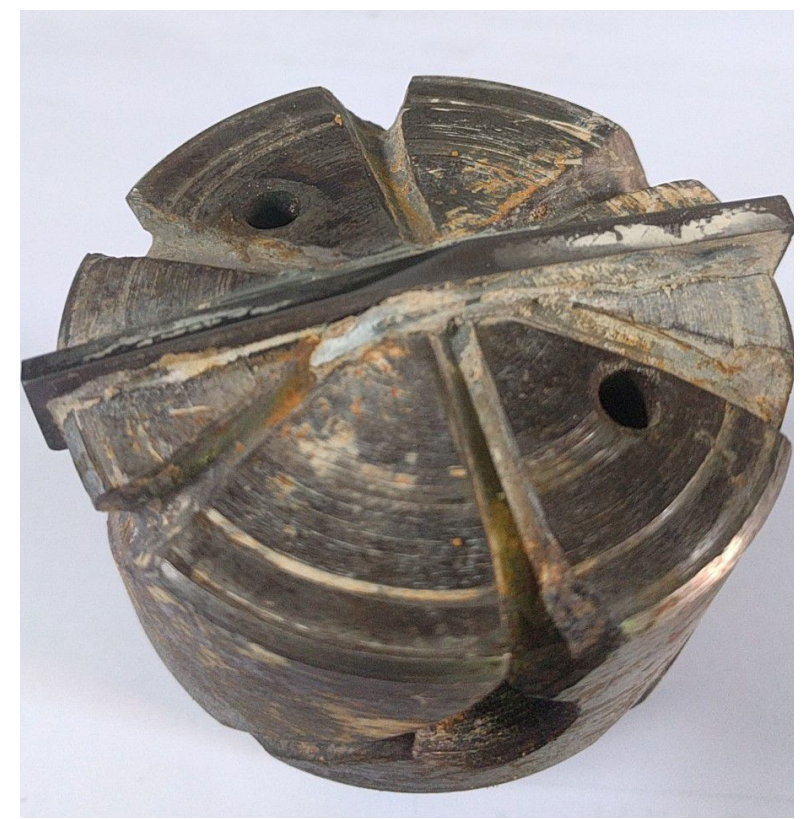

b.

Figura 1. a. Sistema completo de perforación con control de velocidad, central hidráulica, y herramienta de perforación. b. Detalle de la broca con cuerpo realizado en SAE 4140, dos filos de carburo de tungsteno y salidas de fluido.

Fuente: elaboración propia.

a.

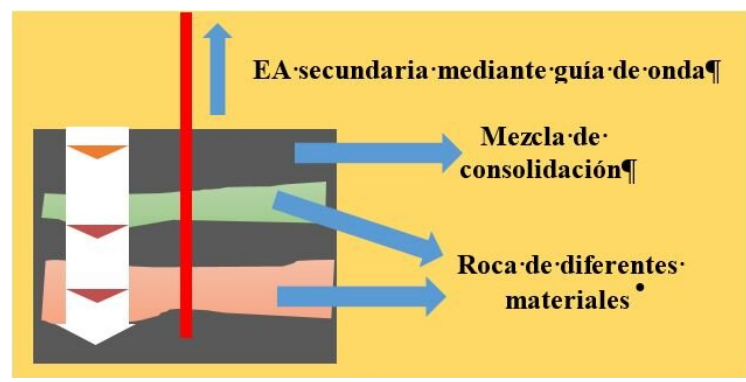

Figura 2. a. Croquis de la composición de las probetas donde se observan los diferentes estratos y la guía de onda que los atraviesa. b. Probeta con cuatro perforaciones y guía de onda cementada.

Fuente: elaboración propia.

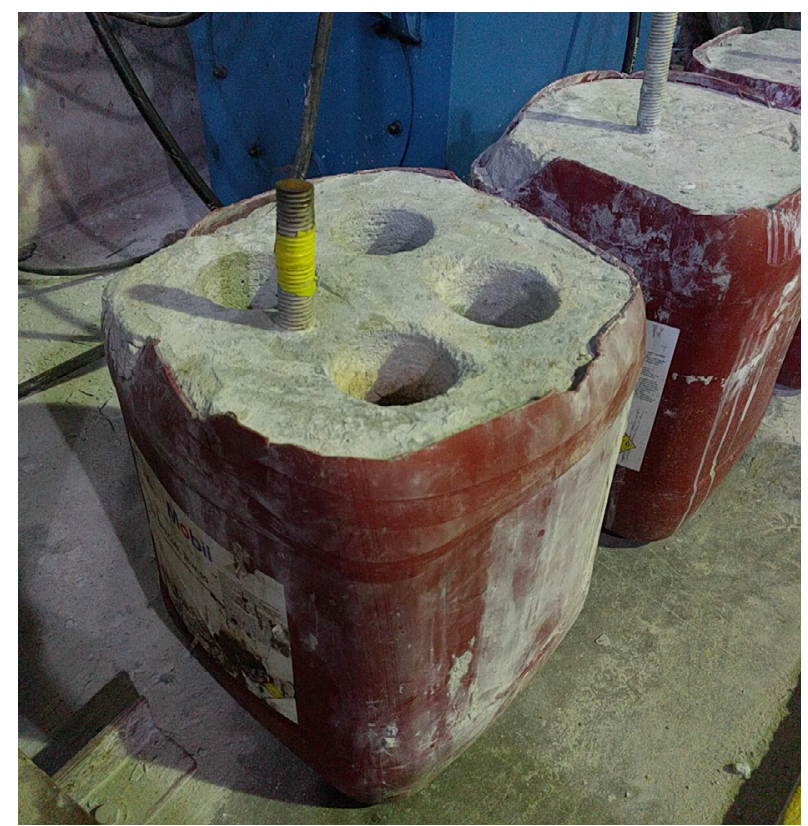

b. 
a.

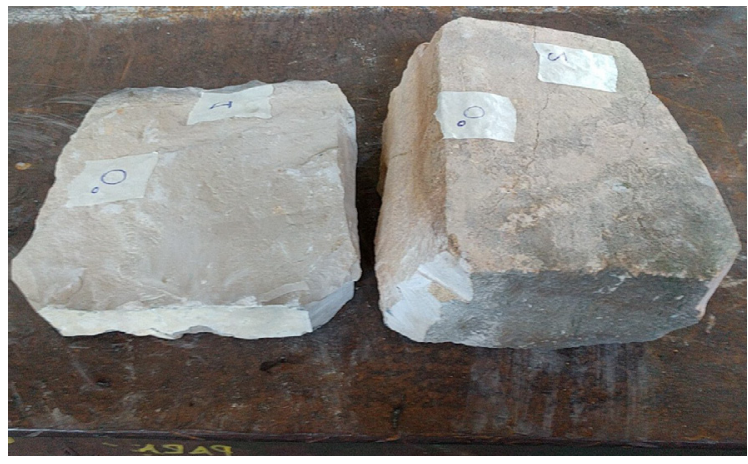

c.

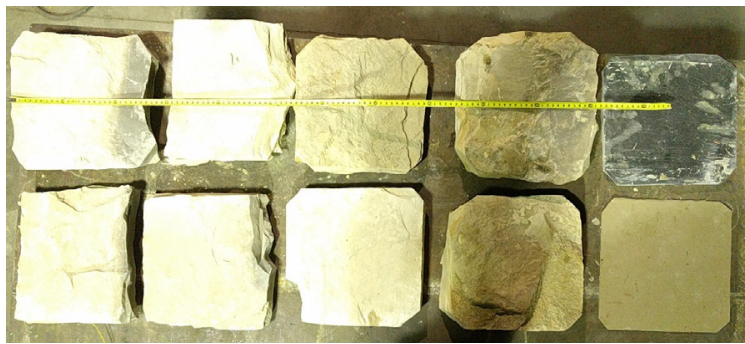

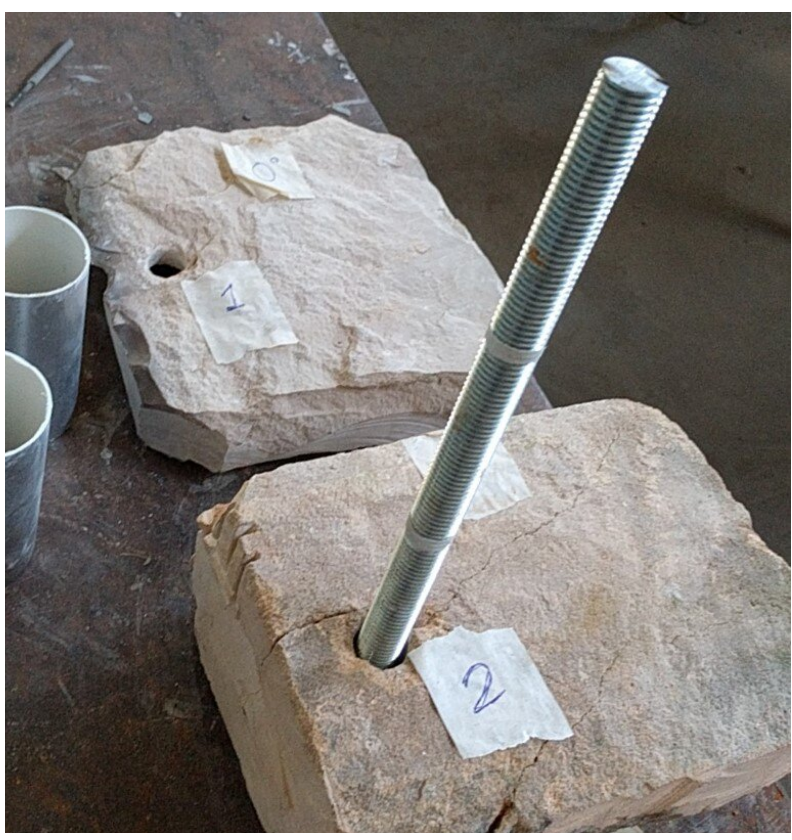

b.

Figura 3. a. Dos tipos diferentes de estratos con variación apreciable de espesor. b. Estratos con perforación para guía de ondas colocada. c. Diferentes estratos utilizados para ensamblar las probetas (arenisca, caliza y pizarra) y su tallado para montaje dentro de cada probeta

Fuente: elaboración propia.

determinado como arenisca, debido a su tamaño de grano, comparable con la sílice y observable a ojo desnudo. Este produce una fractura rugosa que, en general, se propaga por el material de unión de sus granos.

La segunda muestra utilizada está dentro de las Ilamadas lutitas, siendo específicamente esta una caliza. Son rocas sedimentarias de grano más fino que las areniscas, y esta en particular, se compone mayoritariamente por material producto de la sedimentación de moluscos marinos. Este tipo fractura en forma de escama paralela al sentido de deposición de sus elementos constitutivos. Debido a que es más densa que la arensica, si bien es menos dura que esta, es de más difícil taladrado debido al tamaño y densidad en la deposición de sus minerales.

La muestra M2 consiste en una composición de material cementicio con dos capas alternadas de roca metamórfica denominada pi- zarra; es densa (tamaño de grano muy fino), con placas de mica alineadas en sentido paralelo a la laminación. A su vez, se fractura en planos de debilidad paralelos a la laminación, con fracturas suaves o sedosas.

Sistema de emisión acústica: Para la adquisición y procesamiento de los datos se utilizó un sistema de EA de la marca Physical Acoustics Corporation (PAC), modelo DISP-4 de 4 canales, con capacidad de adquisición

Tabla 1. Probetas ensayadas con rocas sedimentarias y metamórficas

\begin{tabular}{|c|c|c|c|c|}
\hline & $\begin{array}{l}\frac{\mho}{U} \\
\frac{\mathscr{n}}{\frac{1}{d}} \\
\frac{1}{<}\end{array}$ & $\frac{\stackrel{\widetilde{N}}{\widetilde{V}}}{\mathcal{U}}$ & 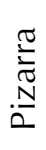 & 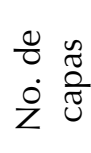 \\
\hline $\begin{array}{l}\text { Muestra } 1 \\
(\mathrm{M} 1)\end{array}$ & $X$ & $X$ & - & 2 \\
\hline $\begin{array}{l}\text { Muestra } 2 \\
\text { (M2) }\end{array}$ & - & - & $X$ & 2 \\
\hline
\end{tabular}

Fuente: elaboración propia. 
de parámetros característicos de la EA y formas de onda. Las señales fueron tomadas con dos sensores (uno en la probeta y otro en la broca) marca PAC modelo WD de banda ancha, con preamplificadores externos modelo 2/4/6 con ganancia ajustada en $20 \mathrm{~dB}$ para el sensor montado sobre la broca, y en 40 $\mathrm{dB}$ para el sensor montado en la probeta. El equipo se manejó en una PC con el software $A E$ Win. Entre los parámetros medidos se registraron: amplitud, RMS, energía MARSE, cuentas, rise time, frecuencia media, centroide de frecuencia, duración, etc.

\section{Arquitectura de red neuronal utilizada para} análisis de probeta M2: La arquitectura de la red neuronal Kohonen creada consta de 400 neuronas dispuestas en forma de grilla toroidal de $20 \times 20$ neuronas, con una cantidad de cuatro vecinos por neurona y una función topológica de ajuste de vecindad tipo gaussiana. El criterio de la neurona ganadora se definió así: "la entrada más parecida al peso de una neurona resulta como ganadora", con ajuste de pesos por datos y por época, y con una duración de entrenamiento de 400 épocas. El error de mezclado y de norma alcanzado en la etapa de entrenamiento fue del $5,9 \%$ y 0,09 , respectivamente. La red fue entrenada y simulada con un vector dato (VD) multiparamétrico de 5 componentes [Amplitud, energía MARSE, RMS, rise time, frecuencia promedio], extraído de la señal de EA de la broca y proveniente de la perforación. La cantidad de VD utilizados fue de 400 para el entrenamiento y 196 para la predicción. Estos VD fueron seleccionados gráficamente respecto de las señales de EA, pertenecientes a la perforación de las dos rocas de pizarra (513 VD) y al material cementicio (83 VD) para la probeta $\mathrm{M} 2$.

Desarrollo del ensayo: Las probetas M1 y M2 fueron taladradas en cuatro sectores, tomando señales representativas de cada proceso. El tiempo de taladrado en estos ensayos en particular promedió los 1800 s. La EA se adquirió de manera paralela sobre la herramienta (canal 1) y la probeta (canal 2), con el fin de comprar señales y comprobar el correcto funcionamiento de la instrumentación de la herramienta giratoria de perforación. Una vez taladradas las probetas M1 y M2, se analizó la información parametrizada. En la probeta M1 se caracterizaron las zonas de las interfaces respecto de las diferentes rocas taladradas, mientras que para la probeta M2 se utilizaron algunos de los parámetros de EA para entrenar y simular mediante una RNK, luego de un posprocesamiento de la señal que comprende selección de los datos, etiquetado y normalización, además del análisis de correlación entre variables.

\section{RESULTADOS}

Para la probeta $M 1$, en la figura $4 a, b, c, d, e, f$. se ilustran los parámetros amplitud [dB], energía MARSE [ $\mu \mathrm{v} . s / c u e n t a s]$ (área medida respecto de la evolvente de la señal rectificada de EA) y RMS [v] de la EA para los canales 1 y 2. Se observa que el sistema montado directamente sobre la herramienta de trabajo recibe una potencia de señal de mayor amplitud (6 a 8 veces) que la señal obtenida a través de la probeta. Esto posiblemente sea debido a que las ondas ultrasónicas encuentran en el metal del cuerpo de la broca una conexión directa con el proceso y un camino con mucha menor atenuación que a través de las interfaces de la roca, cuyo material es más dispersivo y atenuante. El análisis de la amplitud muestra que es bastante difícil detectar las variaciones entre diferentes zonas de estas probetas compuestas por estos tres elementos. Por otro lado, los parámetros de energía MARSE y el valor RMS de la EA muestran zonas de variación más marcadas respecto de la amplitud para diferentes partes del ensayo de la muestra M1. 
Estas variaciones pueden ser relacionadas (mediante análisis del material cortado y posición de la punta de la herramienta) con las correspondientes interfaces rocosas contra
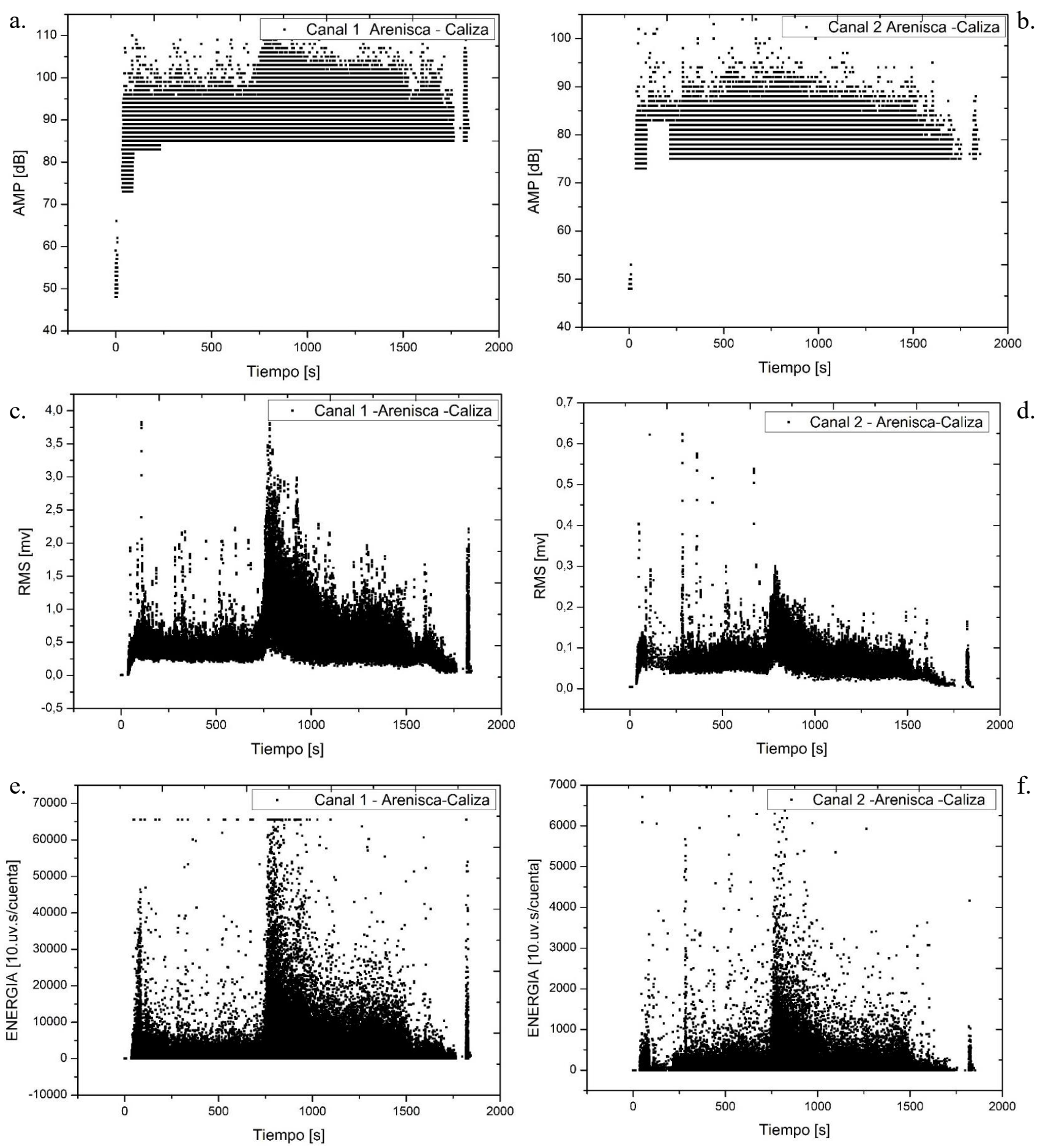

Fuente: elaboración propia.

las cuales se fue encontrando el cortador de carburo. Estas interfaces están marcadas en la figura 5. y corresponden a:

Figura $\mathbf{4 a}, \mathbf{b}, \mathbf{c}, \mathbf{d}, \mathbf{e}, \mathbf{f}$, . Muestra una marcada variación de los parámetros de EA en función de los estratos perforados. a y b. Parámetro RMS de EA medido sobre el canal 1 (broca de perforación) y canal 2 (guía de onda colocada dentro de la probeta). c y d. Parámetro de energía MARSE para canales 1 y 2, respectivamente 
Zona 1: inicio del proceso de taladrado sobre material de cementación de la probeta.

Zona 2: fin de zona 1 e inicio del taladrado de roca tipo arenisca.

Zona 3: fin de elemento cementación e inicio de taladrado de roca tipo caliza.

Estas zonas características de inestabilidad se observan cuando la broca hace un cambio de interfaz entre diferentes materiales geológicos y se pueden relacionar al progresivo avance del cortador dentro del nuevo material que comienza a cortar.

Al inicio de cambio de interfaz, la velocidad de corte de la punta de la broca es cero (0) y crece radialmente hacia el diámetro de esta, de manera que el primer contacto entre el cortador y la nueva interfaz se produce casi por indentación, más que por corte del material. Una vez que el cortador ingresó plenamente dentro del nuevo material, el corte se hace más estable y equilibrado, lo cual disminuye la tasa de emisión y la energía de la señal, y baja su amplitud máxima.

La comparación entre los canales 1 y 2 indicaría que la atenuación producida por el sustrato rocoso actúa como un filtro natural; así se disminuye la intensidad de la señal, en general, y las de alta frecuencia, en particular, ya que la atenuación es función creciente con la frecuencia. Esto permite tener una

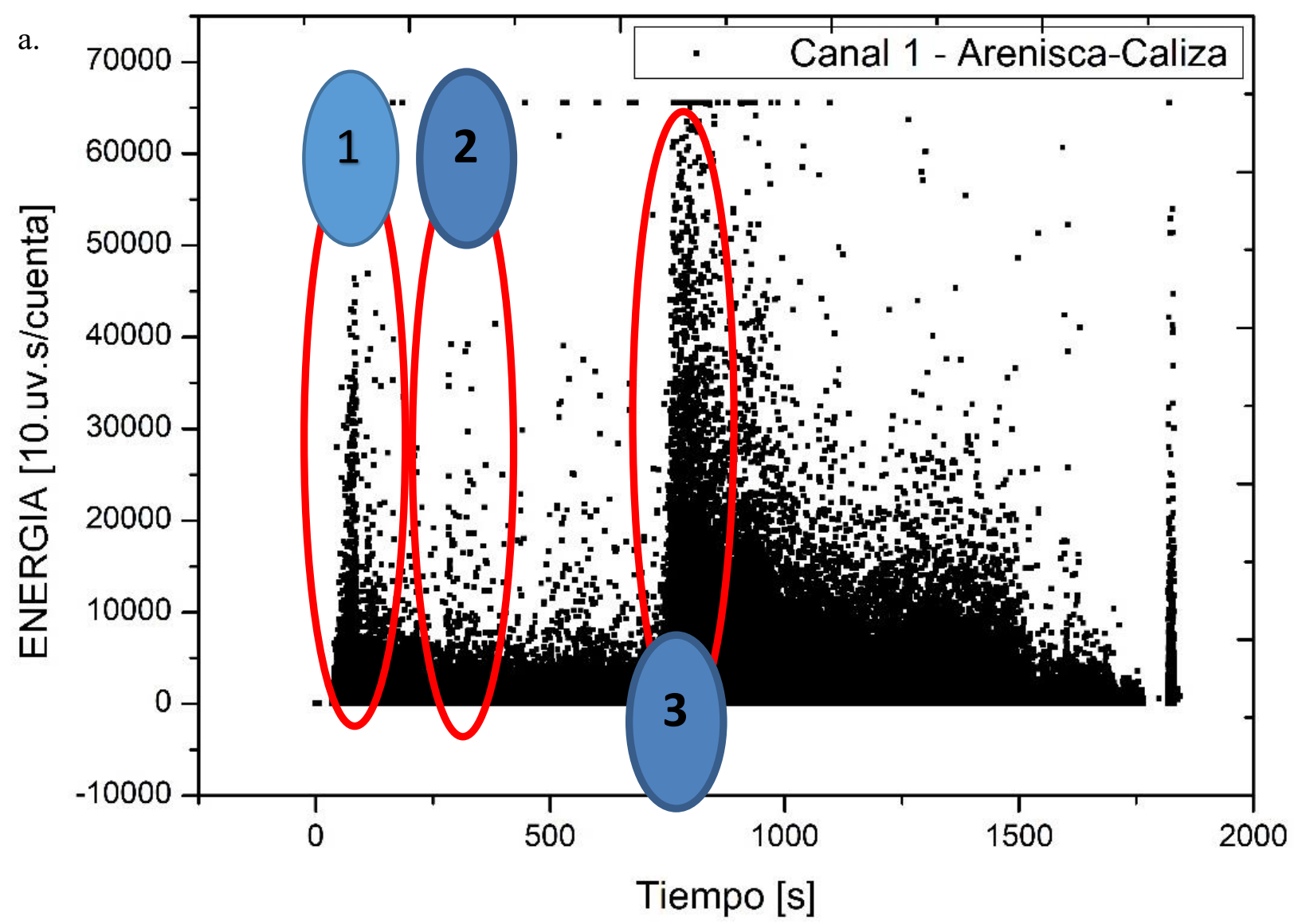




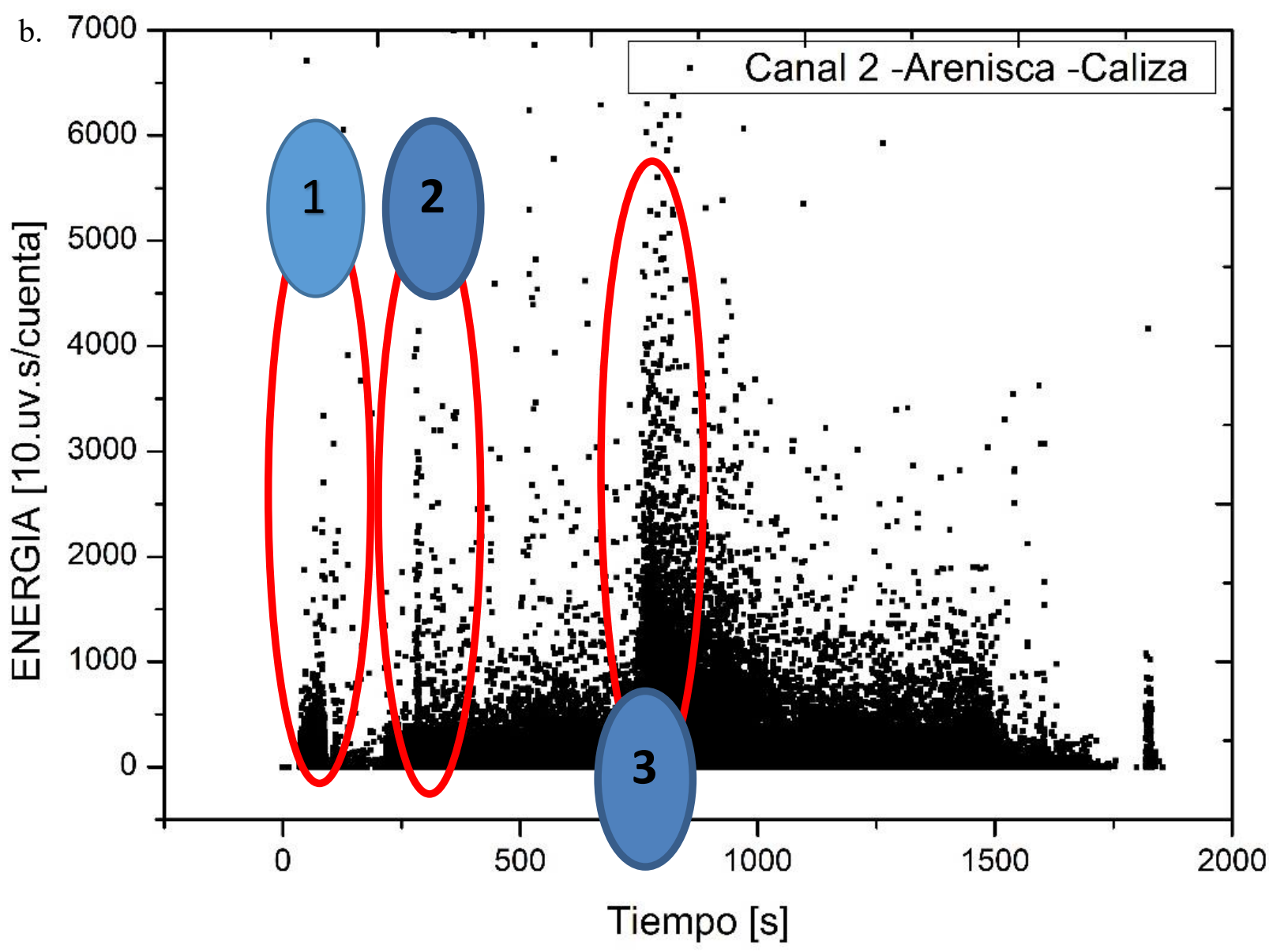

Figura 5. Marcación de zonas de interfaz identificadas mediante a. Energía MARSE para canal 1. b. Energía MARSE para canal 2

Fuente: elaboración propia.

señal más limpia en la que se observan los cambios de interfaz de manera más clara. En contrapartida, parte de la información recibida por este canal puede ser perdida durante este efecto de filtrado natural.

Aquella proveniente de la herramienta de corte se muestra altamente correlacionada con la de la guía de onda de la probeta, mostrando que este canal de medición es representativo de las variaciones del proceso, con la ventaja de que no se necesita colocar una guía de onda por cada probeta a ensayar, ya que la cadena de medición es siempre la misma.
Para la probeta M2 - conformada por dos capas de roca pizarra cementadas-, también se pueden detectar estas interfaces, a medida que con la perforación se atraviesan los diferentes sustratos (figura 6).

La señal de EA proveniente de la instrumentación de la herramienta (canal 1) fue seleccionada en función de los gráficos para el proceso de entrenamiento y predicción de la RNK (figura 6). Estos tramos de información se etiquetaron como pizarra uno (P1), para el primer estrato de pizarra; pizarra dos (P2), para el segundo estrato, y finalmente, el elemento de consolidación, que se encuentra sobre, debajo y en medio de 
las capas, se nombró cementicio (C). Estas etiquetas sirvieron para evaluar el desempeño de la red en la clasificación, durante el entrenamiento y posteriormente en la predicción.

Los resultados del mapa de entrenamiento de Kohonen de la figura $7 \mathrm{a}$. muestran una separación en clases de las señales pertenecientes al material $C$, respecto de los estratos de pizarra P1 y P2. Adicionalmente, la red tuvo la capacidad de diferenciar las señales provenientes de las dos rocas del mismo tipo de material metamórfico (P1 y P2), pero de diferente profundidad. Los resultados mostrados en la figura $7 \mathrm{~b}$. corresponden a la simulación realizada en el mapa ya entrenado.

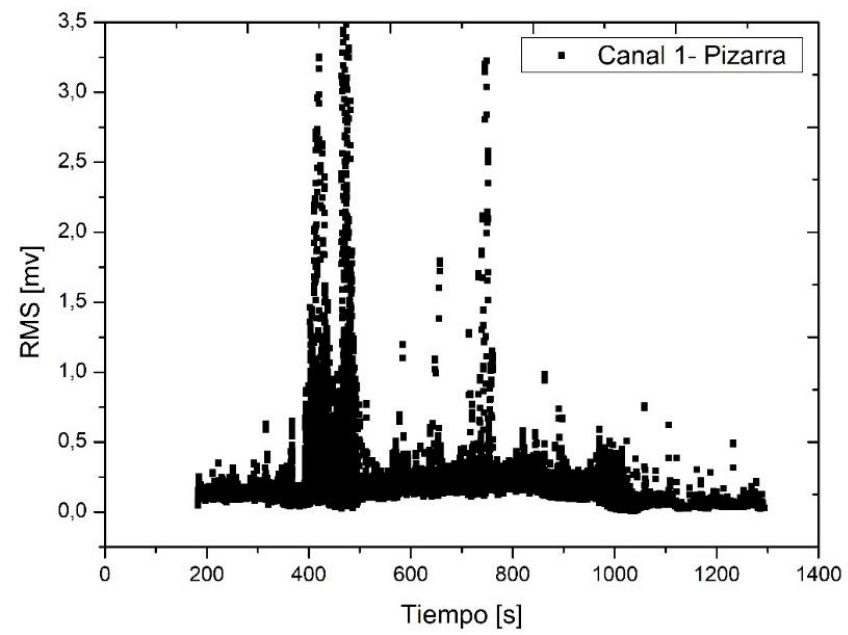

Estos datos nunca fueron procesados por la red hasta el momento de la simulación, y fueron clasificados con un error de mezclado y de norma inferior al $5 \%$ y 0,04 , respectivamente. Se respetan las zonas topológicas delimitadas por las neuronas que clasifican los datos de las señales $C$ respecto de las de P1 y P2. También se observa la formación de subclúster entre P1 y P2, a pesar de ser el mismo material.

\section{CONCLUSIONES}

Los resultados indican que la EA tiene la capacidad de proveer información útil para distinguir diferentes materiales geológicos

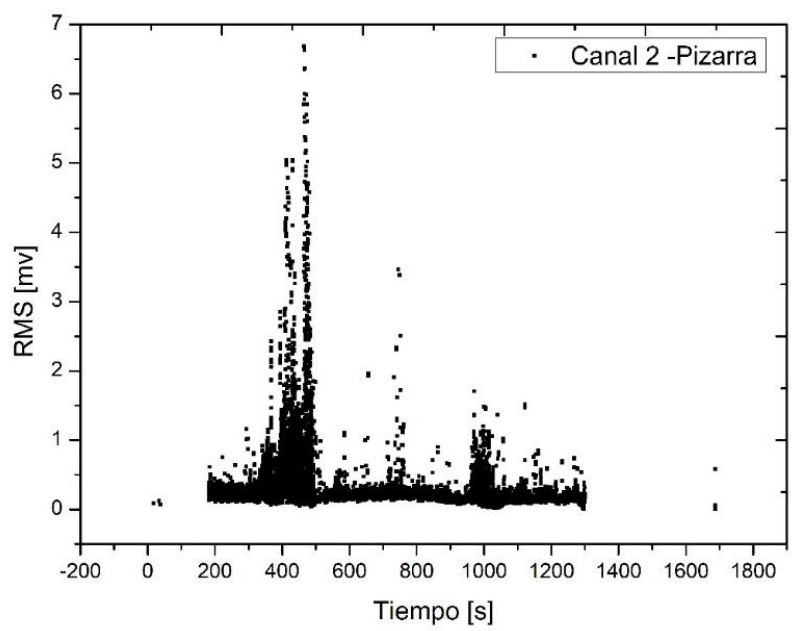

Figura 6. Medición del parámetro RMS de la EA para probeta M2 de roca pizarra, canal 1 y canal 2, respectivamente. Fuente: elaboración propia. 
a.

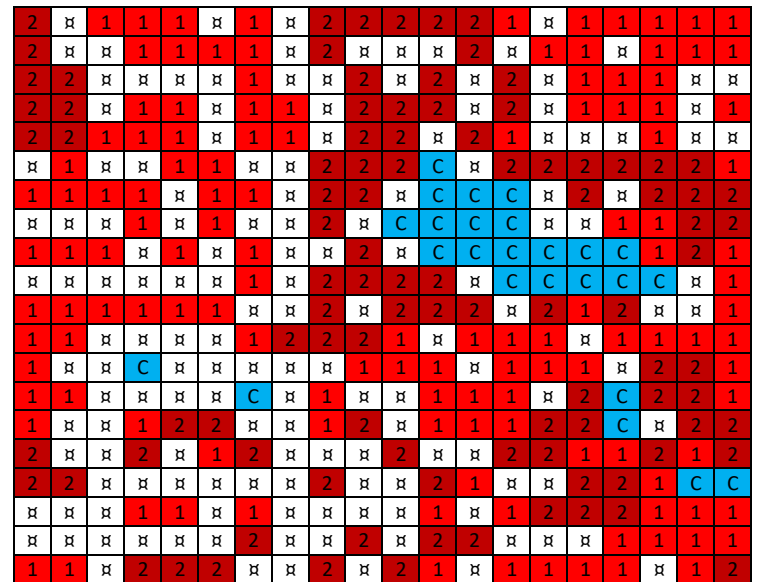

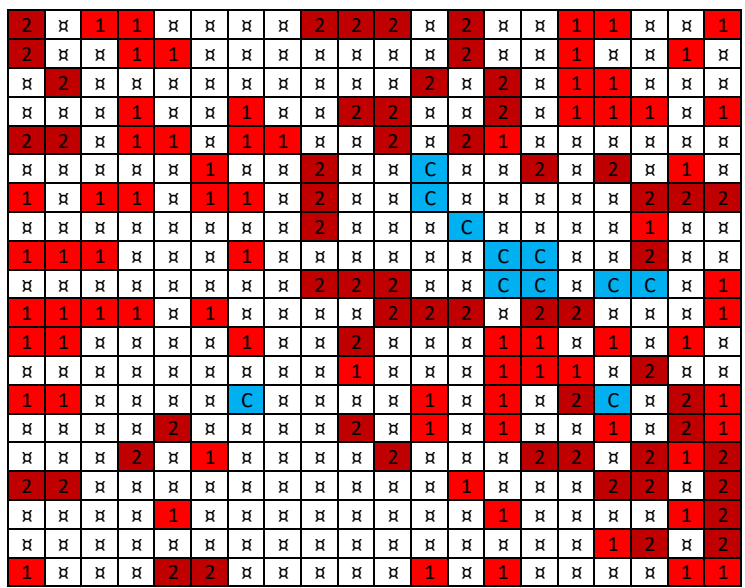

b.

Figura 7a. Mapa de Entrenamiento de RNK para probeta M2 donde figuran P1, P2 y C.

Figura 7b: Mapa de simulación y clasificación para probeta M2 con datos desconocidos por la RNK

Fuente: elaboración propia.

durante el proceso de perforación a esta escala. El parámetro de amplitud máxima de la EA no muestra claramente los cambios de la dinámica de corte que se suceden en los estratos, mientras que la energía MARSE y el RMS de la señal muestran transitorios relacionados con el cambio de material que se está perforando, posiblemente debido a la progresiva penetración del cortador en este.

La composición intrínseca de la probeta de material geológico filtra naturalmente la señal de EA, produciendo que las señales registradas por la guía de ondas dentro de la probeta sean de menor amplitud que las registradas directamente sobre la broca. Ambas señales mostraron una alta correlación de eventos, lo cual valida la utilización de la instrumentación sobre la broca como método para el monitoreo del proceso.

Con la información paramétrica de EA proveniente de la broca se logró entrenar y simular una RNK que mostró capacidad de clasificar la información perteneciente a cada tipo de material por separado, con un error de mezcla inferior al $5 \%$.

\section{FINANCIAMIENTO}

Esta investigación se desarrolló en el marco de una tesis de doctorado avalada y financiada por la Universidad Tecnológica Nacional (UTN) de la República Argentina.

\section{AGRADECIMIENTOS}

Se agradece a la empresa CARSOL S.R.L. por su valiosa colaboración y aporte en la construcción del prototipo de perforación.

\section{REFERENCIAS}

Barrera, G., Carreón, G. y Ruiz, A. (2005). Control de calidad de piezas cerámicas por medio de análisis de vibraciones y redes neuronales artificiales. Computación y Sistemas, 8, 187-195. 
Bourgoyne Jr., A. et al. (1991). Applied drilling engineering. Richardson, EE. UU.: Society of Petroleum Engineers.

Chen, Y. y Liao, Y. (2003). Study on wear mechanisms in drilling of Inconel 718 superalloy. Journal of Materials Processing Technology, 140, 269-273.Dornfeld, D. y Kannatey-Asibu, E. (1980). Acoustic emission during orthogonal metal cutting. International Journal of Mechanical Sciences, 22, 285-296.

Everson, C.E. y Cheraghi, S.H. (1999). The application of acoustic emission for precision drilling process monitoring. International Journal of Machine Tools and Manufacture, 39, 371-387.

Godin, N., Huguet, S. y Gaertner, R. (2005). Integration of the Kohonen's self-organising map and k-means algorithm for the segmentation of the $\mathrm{AE}$ data collected during tensile tests on cross-ply composites. NDT\&E International, 38(4), 299-309.

Gómez, M.P. (2012). Estudio de las señales de EA generadas en el proceso de corte de metales. Aplicaciones a procesos de taladrado. [Tesis de doctorado]. Universidad Nacional de General San Martín (UNSAM). Buenos Aires, Argentina.

Gómez, M.P., Hey, A.M., D'Attelis, C.E. y Ruzzante, J.E. (2012). Assessment of cutting tool condition by acoustic emission. Procedia Materials Science, 1, 321-328.

Gómez, M.P., Hey, A.M., Ruzzante, J.E. y D'Attellis, C.E. (2010). Tool wear evaluation in drilling by acoustic emission. Physics Procedia, 3, 819-825.
Grabec, I. y Leskovar, P. (1977). Acoustic emission of a cutting process. Ultrasonics, 15(1), 17-20.

Jemielniak, K. y Otman, O. (1998). Tool failure detection based on analysis of acoustic emission signals. Journal of Materials Processing Technology, 76(13), 192-197.

Kohonen, T. (2001). Self-Organising maps. Heidelberg: Springer Verlag.

Mascaro, B., Gibiat, V., Bernadou, M. y Esquerre, Y. (2005). Acoustic emission of the drilling of carbon/epoxy composites. Paper presented at the Forum Acusticum. Budapest, Hungría.

Peng, S. y Zhang, J. (2007). Engineering geology for underground rocks. Heidelberg: Springer-Verlag Berlin Heidelberg.

Philippidis, T., Nikolaidis, V. y Anastassopoulos, A. (1998). Damage characterization of carbon/carbon laminates using neural network techniques on $\mathrm{AE}$ Signals. NDT\&E International, 31(5), 329-340.

Rajabov, V. et al. (2012). The effects of back rake and side rake angles on mechanical specific energy of single PDC cutters with selected rocks at varyng depth of cuts and confining pressures. En IADC/SPE Drilling Conference and Exhibition (pp. 205-222). California, Estados Unidos.

Saini, D. y Park, Y. (1996). A quantitative model of acoustic emission in orthogonal cutting operations. Journal of Materials Processing Technology, 58, 343-350. 
Zupang, J. y Gasteiger, J. (1993). Neural networks for chemists. An introduction. Nueva York: $\mathrm{VCH}$, Weinheim.

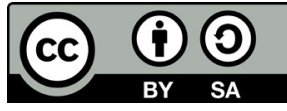

